\title{
Ch. Delsol, J. Nowicki (éd.), La vie de l'esprit en Europe centrale et orientale depuis 1945. Dictionnaire encyclopédique, Les Éditions du Cerf, Paris 2021, pp. I000.
}

Intermittente: così si potrebbe definire l'interesse che, da circa un secolo, la Francia dimostra nei confronti dei paesi dell'Europa centro-orientale. Un'intermittenza spesso condizionata da considerazioni ideologiche o politiche.

Dopo la prima guerra mondiale, la nascita o l'ingrandimento, a scapito degli imperi centrali e della Russia zarista, di un gruppo di stati centro ed esteuropei - Cecoslovacchia, Polonia, Jugoslavia, Romania - protetti dalla Francia e da essa considerati altrettanti alleati in caso di un nuovo conflitto con la Germania suscitò una prima ondata di curiosità per quell'area. La creazione di riviste interamente o parzialmente dedicate all'Europa centro-orientale ("Le monde slave" [1917], la "Revue des études slaves" [192I]), l'istituzione di cattedre di polacco e ceco all'École des Langues orientales (I92I) costituirono alcune manifestazioni di quest' interesse.

Il fallimento della politica estera francese, la guerra e, soprattutto, la sovietizzazione di quasi tutti i territori situati ad est dell'Elba cambiarono radicalmente la situazione. L'Europa centrale e orientale appariva ormai un blocco uniforme controllato da Mosca e di cui, all'infuori degli addetti ai lavori, si sapeva in fondo ben poco. Gli anni '8o segnarono una svolta nella visione francese di quell'area. Fu determinante in questo senso un saggio di Kundera, pubblicato nel 1983 nella rivista "Le débat": secondo l'autore de Lo scherzo, l'Europa centrale - il romanziere ceco limitava la sua analisi alla Polonia, all'Ungheria e al proprio paese di origine - non era l'avamposto del sovietismo bensì un Occidente "kidnappé", artificialmente strappato dalla storia recente al resto dell'Europa. Nel clima di speranza suscitato dalla perestrojka e sulla scia del saggio kunderiano, varie riviste si assunsero il compito di esplorare quel continente dimenticato: "Lettre internationale", "L'autre Europe" (entrambe nate nel 1984), "Le messager européen” (1987). Da queste pubblicazioni emergeva l'immagine di un'Europa centroorientale serbatoio di valori umanisti - libertà, rigetto della violenza, protezione dei diritti dell' individuo -, ma anche avversa ad ogni nazionalismo e dotata di una buona dose di umorismo dissacrante e di autoironia: Kafka, Hašek e Kiš figuravano fra i numi tutelari di "Lettre internationale”. Contemporaneamente, man mano che si sgretolava l'impero sovietico, l'editoria francese licenziava ogni anno un numero sempre crescente di libri provenienti dall'area centro-orientale del continente europeo (vedi Ioana Popa, Traduire sous la contrainte. Littérature et communisme - 1945-1989, [2010]). La guerra e lo scatenarsi dei nazionalismi nell'ex Jugoslavia segnarono una battuta d'arresto. Tutte le riviste soprammenzionate smisero le pubblicazioni negli anni ' 90 e, senza abbandonarli del tutto, le case editrici francesi si disinteressarono degli autori centro e esteuropei. 
Alle soglie del 2000 , l'integrazione nell'Unione europea di vari paesi dell'ex blocco sovietico risvegliò la curiosità francese per quell'area. Risveglio ben timido in realtà e la cui manifestazione principale consistette, un anno dopo il primo allargamento, nella pubblicazione del libro di Alexandra Laignel-Lavastine, Esprits d'Europe (2005). Specialista della Shoah e buona conoscitrice della Romania novecentesca (Cioran, Eliade, Ionesco: l'oubli du fascime. Trois intellectuels roumains dans la tourmente $d u X X^{e}$ siècle, 2002), l'autrice vi esaminava il pensiero antitotalitario ma anche antinazionalista di tre grandi intellettuali centroeuropei: Miłosz, Patočka e Bibó.

Con la summa pubblicata a cura della filosofa Chantal Delsol e di Joanna Nowicki, la ricezione francese delle culture centro e esteuropee sembra essere entrata in una quarta fase. Nuova epoca, sì, ma non meno legata delle precedenti alle circostanze particolari del momento in cui è stata inaugurata, cioè di un momento in cui, agli occhi di molti francesi, i paesi del centro e dell'est del continente europeo stanno cambiando status e, da alleati potenziali (fase I), modelli rivoluzionari antitotalitari (fase 2 ) o meri partner economici (fase 3 ), sono diventati i protagonisti di una rivoluzione conservatrice. Sembra infatti che i dissidenti di una volta siano stati sostituiti dagli inquietanti portavoce di un populismo illiberale. Per molti cittadini dei paesi più ricchi dell'Ue, l'Europa centrale e orientale ha ormai il volto di un Orbán o di un Kaczyński e non suscita quasi altro che diffidenza o, semmai, indifferenza. Una situazione inaccettabile per Chantal Delsol e Joanna Nowicki che, con il loro dizionario enciclopedico, hanno voluto "participer au rétablissement des ponts rompus par le silence, la méconnaissance ou les préjugés mutuels” ( $\mathrm{p}$. 23). Per le due autrici in effetti non c'è nessun dubbio: se, per più di $40 \mathrm{anni}$, le nazioni europee sono state divise dalla guerra fredda o se, ancora oggi, esprimono pareri diversi su alcune questioni (l'immigrazione, l'aborto), procedono tutte da una stessa matrice "marquée par la culture de liberté issue du judéo-christianisme et de la révolution des Droits de l'homme” (p. Is). Una migliore comprensione reciproca è dunque possibile.

Per attuare un tale proposito, le voci del dizionario sono state suddivise in due parti. La prima, enciclopedica, passa in rassegna tematiche che hanno alimentato il dibattito intellettuale in vari paesi dell'Europa centro-orientale dal 1945 ai nostri giorni (pp. 27-535). Precisiamo subito che, per ragioni materiali, le curatrici del volume non hanno preso in considerazione tutti i territori interessati operando scelte talvolta sorprendenti. Così, malgrado la loro evidente appartenenza all'area studiata, le componenti dell'ex Jugoslavia sono state escluse dalle trattazioni, mentre l'Estonia, sebbene si consideri generalmente più nordeuropea che esteuropea, è ben presente nel libro.

Ciononostante questa parte enciclopedica è ricca di contenuti e affronta argomenti diversi quanto interessanti come Centre(s) et périphérie(s), Environnement et société dans l'Europe centrale après 1945, Nationalismes et minorités, ecc. Benché la maggioranza dei contributi si concentri su tematiche prettamente centro ed esteuropee, questa prima sezione non trascura neanche i transfer culturali svoltisi tra l'Europa centro-orientale e la Francia (Exil francophone, Transfert culturel, Visiteurs français et Hongrie). Se nell'elenco dei contributori compaiono i nomi di alcuni noti specialisti francesi (Maria Delaperrière, Liouba Jurgenson, Xavier Galmiche), le curatrici del volume hanno scelto di affidare la stesura della maggior parte dei lemmi a studiosi centro o esteuropei "pour provoquer un dialogue, en acceptant parfois une vraie différence de style d'approche” (p. 23).

Questo principio vige anche nella seconda parte del libro (pp. 539-932), costituita da circa 260 schede biografiche dedicate a varie personalità centro ed esteuropee: scrittori (Hrabal, Kundera, Škvorecký, Herling-Grudziński, Konwicki, Różewicz, per fare qualche esempio appartenente all'area slava), storici (Lucian Boia, Jenó Szúcs, Bronisław Geremek), filologi e teorici della letteratura (Adrian Marino, Jiři Levý). Tuttavia la categoria più rappresentata in questo repertorio biografico è 
senz'altro quella dei pensatori lato sensu (filosofi, saggisti, teologi). Il libro annunciava del resto sin dal titolo l'intenzione di trattare della “vie de l'esprit" nell'Europa centrale e orientale.

A dir la verità, il tema dell'Europa centrale (e orientale) come 'supplemento d'anima' esiste da tempo. Dagli anni ' 80 , la lettura degli scrittori e pensatori centro ed esteuropei viene regolarmente presentata come un antidoto alle presunte debolezze delle società capitaliste europee: la rinuncia ai valori dell'alta cultura, la politicizzazione del pensiero e la conseguente incapacità degli intellettuali occidentali di veder il vero volto del regime sovietico in Kundera; l'abbandono di ogni idea di Europa e il vuoto burocratico dell' Ue che ne deriva in Laigniel-Lavastine. Da questo punto di vista, il libro qui recensito non è molto diverso dai testi suddetti. Parlando dell'Europa centrale e orientale, intende anche parlare dell'Europa occidentale. Ma lo fa in un modo militante che, a mio avviso, nuoce considerevolmente all'opera. Certo un volume collettivo che coinvolge quasi 200 collaboratori non può offrire una visione unitaria e omogenea del suo oggetto di studio. Nondimeno molte pagine de La vie de l'esprit en Europe centrale et orientale depuis 1945 veicolano un messaggio politico chiaro e coerente. Da questo punto di vista, l'articolo introduttivo di Chantal Delsol è emblematico. Autrice di varie opere in cui difende posizioni alquanto conservatrici, la filosofa sostiene che "pour comprendre l'Europe centrale, il faut faire appel non seulement au logos et à la raison occidentale bien maniée, mais aussi aux mystères abyssaux dont se nourrit toute culture, aux mythes et aux symboles qui en portent les significations. Ainsi les Centre-Européens ont-ils souvent l'impression qu'aux Occidentaux, il manque pour ainsi dire une case, celle qui relie au sacré, car il n'y a d'homme complet que sous l'horizon du mystère - d'où le sentiment nourri par certains d'une décadence de l'Occident" (pp. 22-23). Un sentimento condiviso dalla stessa Delsol che, nell'articolo Démocraties illibérales analizza in questi termini i populismi che dominano oggi la vita politica in alcuni paesi centro ed esteuropei: "Il y a probablement la demande d'une révolution conservatrice, celle qui combat le matérialisme, la décadence des mœurs, l'universalisme excessif, pour défendre l'enracinement, la spiritualité éthique et les identités. Les sociétés d'Europe centrale se rebellent contre une certaine modernité que voudrait leur imposer l'Union européenne” (p. 157). Una modernità - globalizzazione, dissoluzione delle identità, elogio del multiculturalismo, abbandono delle fedi religiose - che costituisce da tempo il bersaglio principale dei suoi libri.

Se è possibile non concordare con tale visione, chiaramente espressa varie volte nel volume, si deve, però, ammettere che essa è plausibile e non si può rimproverare alle curatrici del libro di aver dimostrato una certa soggettività nel ritratto dell'Europa centrale e orientale che emerge da queste mille pagine di articoli e di schede biografiche. Sennonché, in un'opera che ha pretese scientifiche, l'esercizio della soggettività non può fare a meno della massima onestà intellettuale nel trattamento dell' informazione. Non è purtroppo sempre così in questo volume. A furia di voler fare dell'Europa centrale e orientale un 'regno dello spirito' a cui i suoi vicini più occidentali farebbero bene ad ispirarsi, si tende talvolta a trascurare o addirittura a cancellare gli aspetti più imbarazzanti della biografia e dell'opera di alcuni intellettuali indicizzati. Significativa è a questo riguardo la scheda dedicata a Mircea Eliade. Non vi si trova una sola riga sull'impegno dello storico delle religioni nella Guardia di ferro, movimento fascista rumeno violentemente antisemita, sebbene il fatto sia oggi ben documentato. Per fortuna non tutte le schede sono simili a questa, ma tali omissioni non possono che gettare discredito sul contenuto generale del volume.

Segnaliamo infine che il libro avrebbe necessitato di un serio lavoro di editing. Errori ortografici dalle prime alle ultime pagine, nomi propri mal riportati (Giedroyc sistematicamente scritto Giedroyć nell'articolo intitolato "Giedroyć [sic]. Les écrivains du cercle de Kultura"; Jan Zábrana chiamato ora Zadrana ora Zrana nell'articolo sull'Incommunicabilté d'expériences Est/Ouest), frasi 
dalla sintassi incerta, riferimenti bibliografici mancanti (come nell'interessante saggio di Michel Maslowski, "Europe du centre-est, Europe médiane, Europe centrale... Tribulation [sic] du terme et de ses connotations"): tutto questo nuoce alla qualità dell'insieme e suscita la perplessità del lettore più benevolo.

Peccato perché, nonostante i suoi difetti e il suo parti pris ideologico, questo volume offre un panorama, parziale ma interessante, di più di 70 anni di vita intellettuale centro ed esteuropea.

Laurent Béghin 